\title{
No need to routinely discontinue anticoagulants before dental extractions
}

Evans IL, Sayers MS, Gibbons AJ, Price G, Snooks H, Sugar AW. Can warfarin be continued during dental extraction? Results of a randomised controlled trial. Br J Oral Maxillofacial Surg 2002; 40:248-242

Question: In patients who need anticoagulant therapy and require dental extraction, is it safe to continue with anticoagulation?

Aim To investigate whether people taking warfarin with an international normalised ratio (INR) within the normal therapeutic range require anticoagulants to be stopped before dental extractions.

Design Randomised controlled trial of 117 patients in a hospital environment.

Intervention The control group stopped taking warfarin 2 days before extraction if the INR was $>2$ and the procedure was rebooked for following day. The intervention group continued to take medication. If the INR was $>4$ on the day of the procedure the patient was withdrawn. The use of tranexamic acid mouth-rinse was not permitted. Extractions were performed under local anaesthetic using as atraumatic a technique as possible.

Outcome measure Clinical notes and patient logbooks were used to record any complications.

Results The rate of bleeding complications was higher in the group continuing with anticoagulation but the difference was not significant.

\begin{tabular}{lccc}
\hline & $\begin{array}{c}\text { No. with } \\
\text { continued warfarin }\end{array}$ & $\begin{array}{c}\text { No. with stopped } \\
\text { warfarin }\end{array}$ & $\begin{array}{c}\text { NNT } \\
(95 \% \mathrm{Cl})\end{array}$ \\
\hline $\begin{array}{l}\text { Patients } \\
\text { Patients with bleeding }\end{array}$ & 60 & 54 & \\
$\begin{array}{l}\text { complications } \\
\text { Patients wtih any }\end{array}$ & $15(25 \%)$ & $7(13 \%)$ & $8(4-47)$ \\
$\quad$ complications & $19(32 \%)$ & $8(15 \%)$ & $6(3-57)$ \\
\hline
\end{tabular}

NNT, Numbers needed to treat; $\mathrm{Cl}$, confidence interval.

Conclusions The study shows that dental extractions can be carried out safely in a hospital without stopping anticoagulation if the patient's INR is $<4.1$.

Evidence-Based Dentistry (2002) 3, 100-101. doi:10.1038/ sj.ebd.6400141

Address for correspondence: Mr AW Sugar, Consultant in Oral and Maxillofacial Surgery, Maxillofacial Unit, Morriston Hospital, Swansea, Wales SA6 6NL, UK. E-mail: adrian.sugar@swansea-tr.wales.nhs.uk

\section{Commentary}

In dentoalveolar surgery, the management of the anticoagulated patient is varied, controversial and, frequently, anecdote-based. The crux of the issue is whether to alter perioperative dosing of warfarin sodium for the subject undergoing dentoalveolar procedures. Some practitioners recommend discontinuing warfarin for 2 or more days before the procedure, whereas others recommend discontinuing warfarin and starting heparin and, finally, others support maintaining warfarin therapy during the perioperative regimen.

Regarding dental extractions, the management decision must balance the risk of developing an embolus when the warfarin is discontinued perioperatively versus the risk of significant bleeding following dental extractions if warfarin therapy is maintained perioperatively. Fortunately, there is a growing body of data providing evidence-based support regarding the perioperative management of anticoagulated patients who need tooth extractions. Specifically, the weight of the evidence supports the strategy of not altering perioperative dosing of warfarin when extracting teeth. ${ }^{1}$ This article by Evans et al. provides excellent additional support for this management strategy.

There are some specific issues to consider regarding the perioperative management of the anticoagulated patient. First, an INR should be obtained on the day of the procedure. Anticoagulation levels are notoriously difficult to control. An INR obtained a week ago may be meaningless in terms of the patient's level of anticoagulation on the day of the procedure. Second, local haemostatic measures should be aggressively implemented. Third, the role of systemic measures is unclear. There are some data to support the use of perioperative tranexamic acid. Personally, I have never used this approach because tranexamic acid is not readily available in the United States. Finally, the clinician must balance the bleeding risk of the operation with the INR level. For example, a single extraction when the INR is $<4$ may be accomplished with minimal risk. For more complex procedures, multiple extractions or extraction of single bony impacted wisdom tooth, an INR of $<3.5$ is desirable. For a full mouth extraction or extraction of multiple impacted teeth, the INR should be $<3.0$. 
In the final analysis, regardless of how the warfarin is managed, the overwhelming majority of patients will do well. If warfarin is discontinued perioperatively, there is a risk of a significant complication, an embolus, with an associated increased risk for death. The risk of an embolic episode if perioperative warfarin is discontinued for dental extractions was estimated to range between 3884 and 30414 patients treated per embolic episode. ${ }^{1}$ There is a low but finite risk of death, ie, major complication, if warfarin is discontinued. If warfarin is maintained, there is an increased risk for bleeding, but there are no reports of death, and managing the complication reaches the level of nuisance.
1. Dodson TB. Managing anticoagulated patients requiring dental extractions: an exercise in evidence-based clinical practice. Evidence-based Dentistry 2002; 3:23-26.

Thomas B Dodson Department of Oral and Maxillofacial Surgery, Harvard School of Dental Medicine, Boston, Massachusetts, USA 